\title{
Revista Brasileira de Enfermagem REBEn \\ Adaptação e validação do instrumento "Positions on nursing diagnosis" para a língua portuguesa
}

\author{
Adaptation and validation of the "Positions on nursing diagnosis" \\ tool to Portuguese language
}

\author{
Adapatación y validez del instrumento "Positions on nursing diagnosis" \\ para la lengua portuguesa
}

Diná de Almeida Lopes Monteiro da Cruz

Enfermeira. Professora Titular da Escola de Enfermagem da Universidade de São Paulo.

\section{Alda Aparecida Mastelaro Hayashi}

Enfermeira. Gerente de enfermagem do Hospital Evangélico. Londrina, Paraná. Mestre em Assistência de Enfermagem pela

UFSC.

\section{Ana Paula Vilcinski Oliva}

Enfermeira. Professora Assistente da Universidade Estadual de Maringá. Maringá,

Paraná. Doutoranda da Escola de Enfermagem da USP.

Consuelo Garcia Corrêa

Enfermeira. Professora da Universidade Mont Serrat. Santos, São Paulo. Doutora pela Enfermagem da USP.

Parte de projeto de pesquisa financiado pela FAPESP (02/02345-0)- Escola de Enfermagem da Universidade de São Paulo.
Submissão: $23 / 11 / 2005$

Aprovação: 10/02/2006

\section{RESUMO}

O instrumento Positions on Nursing Diagnosis (PND) foi desenvolvido na língua inglesa para avaliar a atitude de enfermeiros frente ao diagnóstico de enfermagem. Objetivos: adaptar e validar o PND para a língua portuguesa e verificar as associações entre o PND e variáveis selecionadas. A versão brasileira do PND, denominada Posições frente ao Diagnóstico de Enfermagem (PDE), foi obtida por versão, retroversão e comparação entre a original e a retro-versão. $O$ instrumento adaptado foi aplicado a 400 estudantes de enfermagem e enfermeiros. Os escores no PDE foram positivamente correlacionados com uma afirmação geral favorável ao diagnóstico de enfermagem; a análise fatorial permitiu solução com 3 fatores (avaliativo, potência, atividade). Os estudantes de enfermagem mostraram atitudes mais favoráveis ao diagnóstico de enfermagem.

Descritores: Diagnóstico de enfermagem; Estudos de validação [tipo de publicação]; Análise fatorial.

\section{ABSTRACT}

The Positions on Nursing Diagnosis tool (PND) was developed in English to assess nurses' attitudes toward nursing diagnosis. Aims: to adapt and validate the PND to Portuguese Brazilian language; and to verify associations between PND and selected variables. The Brazilian version of PND, named Posições frente ao Diagnóstico de Enfermagem (PDE), was obtained by translation, back-translation, and comparison between the original version and the back-translated one. The PDE was applied to 400 nurses and nursing students. The PDE scores were positively correlated with a general statement favorable to nursing diagnosis; the factor analysis produced 3 factors (evaluative, potency, and activity). The nursing students showed more favorable attitudes toward nursing diagnosis.

Descriptors: Nursing diagnosis; Validation study [publication type]; Fator analysis, statistical.

\section{RESUMEN}

El instrumento Positions on Nursing Diagnosis (PND) fue desarrollado en la lengua inglesa para evaluar la actitud de enfermeros frente al diagnóstico de enfermage. Objectivos: adaptar y validar el PND para la lengua portuguesa y averiguar las asociaciones entre el PND y variables seleccionadas. La versión brasileña del PND, denominada 'Posiciones frente al Diagnóstico de Enfermage (PDE)' , fue obtenida por versión, retro-version y comparación entre la original y la retro-version. El instrumento adaptado fue aplicado a 400 estudiantes de enfermage y enfermeros. Los escores en el PDE fueron positivamente correlacionados con una afirmación general favorable al diagnóstico de enfermage; la análisis fatorial permitió solución con 3 factores (evaluativo, potencia, actividad). Los estudiantes de enfermage mostraron actitudes más favorables al diagnóstico de enfermage.

Descriptores: Diagnóstico de enfermería; Estúdios de validación [tipo de publicación]; Análisis factorial.

Cruz DALM, Hayashi APM, Oliva APV, Corrêa CG. Adaptação e validação do instrumento "Positions on nursing diagnosis" para a língua portuguesa. Rev Bras Enferm 2006 mar-abr; 59(2):-163-7.

\section{INTRODUÇÃO}

A necessidade de se desenvolverem sistemas eletrônicos para os registros em saúde tem desafiado a enfermagem a utilizar sistemas padronizados de linguagem. Os sistemas de linguagem padronizada para diagnósticos de enfermagem começaram a ser discutidos na enfermagem brasileira no final da década de $1980^{(1)}$. Desde então, pouco a pouco, as enfermeiras vêm se apropriando do conhecimento sobre os conceitos diagnósticos.

As atitudes têm importante papel na aplicação de conceitos, contribuindo para a motivação em realizar comportamentos a eles relacionados ${ }^{(2)}$. As atitudes de enfermeiros frente ao conceito de diagnóstico de 
enfermagem podem constituir variável de controle nos estudos sobre o desenvolvimento do raciocínio diagnóstico, sobre a capacitação dos profissionais para o uso de classificação de diagnósticos e também nos estudos que testem estratégias de ensino do processo diagnóstico. Essas questões estão relacionadas não só à adequada aplicação dos diagnósticos de enfermagem na clínica, na pesquisa e no ensino, mas também ao próprio refinamento dos diagnósticos já aceitos e de sua classificação.

Este estudo teve a finalidade de adaptar para a língua portuguesa e analisar as propriedades psicométricas do instrumento Positions on Nursing Diagnosis que foi desenvolvido na língua inglesa por Lunney, Krenz ${ }^{(3)}$.

É possível que instrumentos desse tipo sejam desenvolvidos de acordo com o contexto de cada investigação. No entanto esse processo é extremamente longo e caro e, além disso, corre-se o risco de dispor de instrumentos com validade externa desconhecida e que produzem dados que não podem ser comparados internacionalmente ${ }^{(4)}$. Essas são algumas das razões pelas quais o uso de instrumentos em contextos culturais diferentes daqueles em que foram desenvolvidos é prática freqüente ${ }^{(5,6)}$.

O Positions on Nursing Diagnosis é uma escala que utiliza o diferencial semântico para estimar as atitudes de enfermeiras ou estudantes de enfermagem frente ao diagnóstico de enfermagem.

O diferencial semântico foi desenvolvido por Osgood, Suci e Tannenbaum em 1957 para mensurar atitudes ou crenças ${ }^{(7)}$. Uma escala de diferencial semântico consiste de dois adjetivos opostos por sete pontos eqüidistantes. Solicita-se ao sujeito que selecione o ponto que melhor descreve sua visão sobre 0 conceito que está sendo examinado ${ }^{(7)}$. A lógica subjacente às escalas de diferencial semântico, que é o caso desse instrumento, parte do reconhecimento de que, na linguagem escrita e falada, as características de objetos e idéias são amplamente comunicadas por adjetivos ${ }^{(8)}$. 0 diferencial semântico mede primariamente os aspectos conotativos do significado, isto é, as implicações que o objeto (conceito) em questão tem para o respondente $^{(9)}$.

No instrumento desenvolvido por Lunney e Krenz ${ }^{(3)}$ solicita-se ao respondente que pontue como se sente em relação ao conceito de diagnóstico de enfermagem em 20 duplas de adjetivos. Um dos adjetivos de cada dupla expressa uma disposição favorável ao conceito e o outro expressa uma disposição desfavorável. Cada dupla é separada por sete pontos eqüidistantes e o respondente deve marcar um deles de acordo com a maior ou menor proximidade da sua disposição com um dos adjetivos de cada dupla.

Considerando que as pesquisas quantitativas sobre diagnósticos de enfermagem devem ser pautadas em critérios objetivos de confiabilidade e validade dos instrumentos que utiliza, este estudo teve os objetivos: adaptar para a língua portuguesa e verificar as propriedades psicométricas do instrumento Positions on Nursing Diagnosis (Lunney, Krenz, 1994) (3); analisar associações entre atitudes frente ao diagnóstico de enfermagem e as variáveis: sexo, idade, tempo de formado, categoria do respondente (enfermeiro ou estudante) e grau de contato com diagnóstico de enfermagem.

\section{MÉTODO}

\section{Versão para a língua portuguesa}

Os autores da escala autorizaram a utilização do instrumento para a finalidade de adaptação para a língua portuguesa e os procedimentos envolveram a versão, retro-tradução e comparação do original e retrotradução. Essa comparação foi realizada pelos próprios autores da escala. Vale ressaltar que há diferentes propostas metodológicas para a realização de adaptação de instrumentos de medida ${ }^{(10)}$ e que, até o momento não há dados que comprovem a superioridade de uma ou de outra. A presente proposta metodológica fundamenta-se nas discussões de alguns autores ${ }^{(4,6,10,11)}$.

A versão para o português foi realizada por três enfermeiras bilíngües com experiência em adaptação de instrumentos de medidas. Num primeiro momento essa versão foi realizada independentemente pelos três tradutores e, a seguir, dispondo-se das três versões, os tradutores reuniram-se para realizar uma única versão de consenso. A versão consensual foi discutida com enfermeiras e estudantes de enfermagem para que analisassem a adequação dos termos utilizados emitindo sugestões de ajustes. O número dessas avaliações seguiu o princípio de saturação, isto é, enquanto houve o aparecimento de sugestões ou comentários originais o processo continuou. Essas sugestões foram novamente consideradas pelos três tradutores que, em consenso, fizeram as modificações que julgaram pertinentes. Com esses procedimentos obtivemos a primeira versão para a língua portuguesa do instrumento.

A versão em língua portuguesa foi traduzida para a língua inglesa (retrotradução) por profissional em tradução inglês-português-inglês, com experiência nesse tipo de trabalho para adaptação de instrumentos e que teve suporte de revisor. Nessa fase ele não conheceu o instrumento na língua original. Com esse procedimento dispusemos então da retro-tradução.

A retro-tradução foi apresentada aos autores da escala original para que, comparando original e retro-tradução, julgassem se tinha havido alterações nos significados originais dos componentes do instrumento. Os pareceres foram analisados por um grupo constituído pelos três tradutores que realizaram as versões para o português, pelo tradutor especializado que fez a retrotradução e pelo seu revisor para ajustar a versão em língua portuguesa, pautados nos pareceres dos autores da escala.

Ao término dessa etapa tínhamos disponível o instrumento em língua portuguesa "Posições frente ao Diagnóstico de Enfermagem" (PDE) para ser testado quanto a suas propriedades psicométricas.

Análise das propriedades psicométricas do instrumento

O PDE foi aplicado a amostra de conveniência composta por 400 enfermeiros e alunos de graduação, de instituições de saúde e ensino de cidades nos estados de São Paulo, Rio de Janeiro, Paraná e Minas Gerais. Junto com o instrumento, havia uma ficha para registro de dados pessoais e questões para que os respondentes indicassem o grau de contato que tinham com diagnóstico de enfermagem em termos de aulas assistidas, eventos sobre o assunto, prática clínica com diagnóstico de enfermagem, leitura e pesquisa. Para cada uma das atividades as alternativas de respostas eram: nada $=1$; quase nada $=2$; pouco $=3$ e muito $=4$.

As respostas obtidas de cada respondente em cada um dos 20 itens do PDE poderiam variar de 1 a 7 pontos e a pontuação total, entre 20 e 140 . Os escores foram computados e submetidos a análises estatísticas.

Considerando que esse instrumento se caracteriza como um instrumento de medida referenciada pela norma (norm-referenced measure ${ }^{(9)}$ ), a validade de construto foi avaliada pela correlação entre os escores na escala e as respostas à afirmação geral "Sou favorável aos diagnósticos de enfermagem". As respostas a essa questão foram indicadas numa única escala tipo Likert de sete pontos, ancorada pelas palavras "nada" num extremo (=1), e "muito" no outro (=7). A hipótese era de que haveria correlação positiva entre os escores da escala e os escores das respostas à afirmação geral.

A análise fatorial de componentes principais foi aplicada com o intuito de verificar se o caráter de unidimensionalidade, identificado em estudo estrangeiro(3), se repetiria com amostra de população brasileira.

Da amostra total, 100 enfermeiros responderam o PDE num segundo momento, precedido por um curso de 12 horas sobre diagnóstico de enfermagem e raciocínio clínico. A primeira aplicação do PDE ocorreu imediatamente antes do início do curso e a segunda imediatamente após. A proposta do curso era de sensibilizar os enfermeiros quanto à importância do diagnóstico de enfermagem e quanto à viabilidade de sua aplicação na prática clínica. Os escores de antes e depois do curso foram comparados para testar a hipótese de que haveria melhora dos mesmos, admitindo-se que um aumento nos escores seria indicativo de validade do PDE.

A estimativa de confiabilidade foi obtida pelo coeficiente alfa de Cronbach sobre as respostas da amostra total e também da amostra de 100 enfermeiros participantes do curso. 
O projeto de pesquisa foi aprovado pelo Comitê de Ética da Escola de Enfermagem da Universidade de São Paulo. Para todas as instituições em que os dados foram coletados, foi feita uma solicitação formal e, nos casos em que houve exigência, o projeto foi também submetido comitê de ética local e obteve pareceres favoráveis.

0 termo de consentimento foi apresentado aos sujeitos, individual e pessoalmente, por um dos pesquisadores ou colaboradores que esteve disponível para qualquer esclarecimento desejado, após autorização formal da instituição onde se encontrava o respondente. Todos os participantes assinaram termo de consentimento após esclarecimento.

\section{RESULTADOS}

\subsection{Características da amostra}

De 398 respostas válidas para essa variável, $184(46,2 \%)$ respondentes eram alunos de graduação em enfermagem, matriculados em diferentes semestres, e 214 (53,8\%) eram enfermeiros. A Tabela 1 mostra a distribuição da população estudada segundo local e categoria.

Tabela 1. Freqüência de enfermeiros e alunos de enfermagem segundo local. São Paulo, 2003.

\begin{tabular}{lccccc}
\hline \multirow{2}{*}{ Local/Categoria } & \multicolumn{2}{c}{ Enfermeiros } & \multicolumn{2}{c}{ Estudantes } & Total \\
\cline { 2 - 5 } & $\mathbf{n}$ & $\%$ & $\mathbf{n}$ & $\%$ & válido \\
\hline São Paulo/ Bauru / Santos & 141 & 35,4 & 53 & 13,3 & 194 \\
Itajubá & 13 & 3,3 & 82 & 20,6 & $95^{*}$ \\
Londrina/Maringá & 55 & 13,8 & 26 & 6,5 & 81 \\
Niteroi & 5 & 1,3 & 23 & 5,8 & 28 \\
\hline Total & $\mathbf{2 1 4}$ & $\mathbf{5 3 , 8}$ & $\mathbf{1 8 4}$ & $\mathbf{4 6 , 2}$ & $\mathbf{3 9 8}^{*}$ \\
\hline
\end{tabular}

*duas respostas faltantes.

A atividade atual predominante dos enfermeiros que compuseram a amostra está representada na Tabela 2 . O grau de contato da população estudada com o tema diagnóstico de enfermagem está representado na Figura 1.

A Figura 1 mostra que os contatos mais intensos eram pela leitura e aulas. As menores intensidades de contato receberam maiores freqüências de respostas nas atividades de pesquisa e prática clínica.

\subsection{Escores obtidos no PDE}

A Tabela 3 mostra o resultado da estatística descritiva dos 20 itens do PDE. Como alguns dos itens são apresentados de forma invertida no instrumento, eles foram corrigidos para a apresentação nessa tabela.
Tabela 2. Distribuição dos enfermeiros segundo atividade atual predominante. São Paulo, 2003.

\begin{tabular}{lcc}
\hline \multirow{2}{*}{ Atividade principal } & $\mathbf{n}$ & Freqüência \\
\cline { 2 - 3 } & 143 & $\%$ \\
\hline Assistência & 60 & 68,4 \\
Ensino & 6 & 28,7 \\
Outros & $\mathbf{2 0 9}^{*}$ & 2,9 \\
\hline Total $^{*}$ & & 100,0 \\
\hline
\end{tabular}

${ }^{*}$ cinco respostas faltantes.

As médias dos escores podem variar entre 1 e 7 e quanto mais próximo de 7 mais favorável é a atitude. Observa-se na Tabela 3 que a média dos escores nos 20 itens variou entre 3,7 e 6,2. 0 item que obteve a menor média $(3,7)$ foi o dos adjetivos difícil e fácil. Nos 19 itens restantes as médias variaram entre 4,4 e 6,2.

\subsection{Estimativas de validade do PDE}

As 396 respostas válidas ao item de posicionamento geral frente ao diagnóstico "Sou favorável aos diagnósticos de enfermagem", em que 1 = totalmente falso e 7 = totalmente verdadeiro, resultaram em média $=6,2$ $( \pm 1,2)$ e mediana $=7$. Teste de correlação de Spearman entre esses resultados e as médias de escores nos 20 itens da escala resultou em 0,64 ( $p<0,001)$.

A análise de componentes principais produziu a extração de três fatores, conforme a Tabela 4, com variância explicada de 61,3\%.

As médias dos escores da sub-amostra de 100 enfermeiros participantes do curso sobre diagnóstico de enfermagem foram 102,1( $\pm 14,2)$ antes do curso e $111,0( \pm 14,8)$ depois do curso. A comparação das médias pelo teste $t$ pareado resultou em $t=-13,28$ df 499 e $p=0,00$, mostrando melhora significativa dos escores após o curso.

\subsection{Estimativas de confiabilidade do PDE}

0 alfa de Chronbach para a amostra de 400, com os 20 itens do PDE foi de 0,94; para a sub-amostra de 100 enfermeiros foi de 0,89 antes do curso e de 0,93 depois do curso.

\subsection{Associações entre variáveis}

Não houve associação entre os escores no PDE e o gênero do respondente (Mann-Whitney $p=0,99$ ). Quanto a idade, o índice de correlação de Pearson foi $-0,25(p<0,001)$, mostrando fraca correlação negativa (quanto mais jovens, mais favoráveis ao diagnóstico). Não houve correlação entre os escores no PDE e o tempo de formado dos enfermeiros (Pearson $=0,07 ; p=0,32$ ).

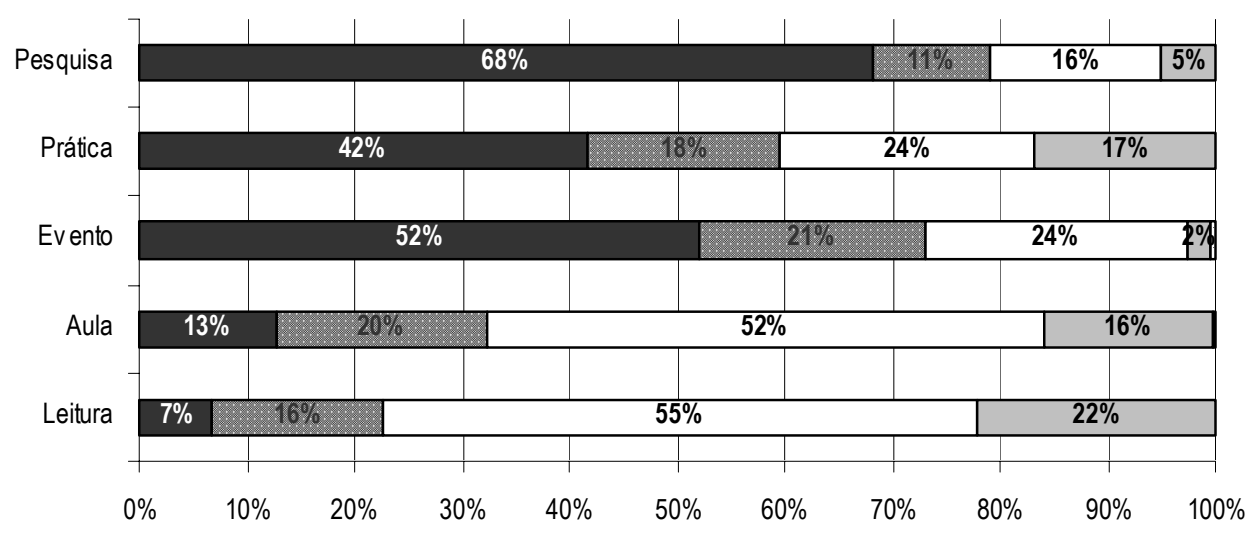

nada quase nada $\square$ pouco $\square$ muito $\square$ missing

Figura 1. Freqüências de respostas nas atividades de contato com diagnóstico de enfermagem segundo intensidade. São Paulo, 2003. 
Tabela 3. Resultados de estatísticas descritivas segundo os itens corrigidos. São Paulo, 2003.

\begin{tabular}{|c|c|c|c|c|c|c|}
\hline Itens & Média & Mediana & Desvio padrão & Mínimo & Máximo & Válidos \\
\hline $\begin{array}{l}\text { Ambíguo }=1 \\
\text { Claro }=7\end{array}$ & 4,2 & 4,0 & 1,6 & 1 & 7 & 79 \\
\hline $\begin{array}{l}\text { Não significativo=1 } \\
\text { Significativo }=7\end{array}$ & 5,7 & 6,0 & 1,2 & 1 & 7 & 80 \\
\hline $\begin{array}{l}\text { Desagradável = } 1 \\
\text { Agradável }=7\end{array}$ & 5,3 & 6,0 & 1,4 & 1 & 7 & 81 \\
\hline $\begin{array}{l}\text { Fraco }=1 \\
\text { Forte }=7\end{array}$ & 5,3 & 6,0 & 1,5 & 1 & 7 & 81 \\
\hline $\begin{array}{l}\text { Sem valor }=1 \\
\text { Valioso }=7\end{array}$ & 6,1 & 7,0 & 1,2 & 1 & 7 & 81 \\
\hline $\begin{array}{l}\text { Negativo }=1 \\
\text { Positivo }=7\end{array}$ & 6,2 & 7,0 & 1,1 & 1 & 7 & 81 \\
\hline $\begin{array}{l}\text { Bobo }=1 \\
\text { Inteligente }=7\end{array}$ & 6,2 & 7,0 & 1,1 & 1 & 7 & 81 \\
\hline $\begin{array}{l}\text { Desconfortável = } 1 \\
\text { Confortável }=7\end{array}$ & 5,0 & 5,0 & 1,5 & 1 & 7 & 81 \\
\hline $\begin{array}{l}\text { Difícil = } 1 \\
\text { Fácil = }\end{array}$ & 3,2 & 3,0 & 1,7 & 1 & 7 & 81 \\
\hline $\begin{array}{l}\text { Não realista }=1 \\
\text { Realista }=7\end{array}$ & 5,4 & 6,0 & 1,4 & 1 & 7 & 80 \\
\hline $\begin{array}{l}\text { Dificultador = } 1 \\
\text { Facilitador }=7\end{array}$ & 5,6 & 6,0 & 1,5 & 1 & 7 & 80 \\
\hline $\begin{array}{l}\text { Inválido }=1 \\
\text { Válido }=7\end{array}$ & 6,2 & 7,0 & 1,1 & 1 & 7 & 81 \\
\hline $\begin{array}{l}\text { Insignificante }=1 \\
\text { Significante }=7\end{array}$ & 6,1 & 6,0 & 1,1 & 1 & 7 & 80 \\
\hline $\begin{array}{l}\text { Irrelevante }=1 \\
\text { Relevante }=7\end{array}$ & 6,2 & 6,0 & 1,1 & 1 & 7 & 81 \\
\hline $\begin{array}{l}\text { Não recompensador }=1 \\
\text { Recompensador }=7\end{array}$ & 5,8 & 6,0 & 1,4 & 1 & 7 & 81 \\
\hline $\begin{array}{l}\text { Inconveniente = } 1 \\
\text { Conveniente }=7\end{array}$ & 5,8 & 6,0 & 1,3 & 1 & 7 & 80 \\
\hline $\begin{array}{l}\text { Inaceitável = } 1 \\
\text { Aceitável }=7\end{array}$ & 6,0 & 6,0 & 1,2 & 1 & 7 & 80 \\
\hline $\begin{array}{l}\text { Ruim }=1 \\
\text { Bom }=7\end{array}$ & 6,1 & 6,0 & 1,0 & 1 & 7 & 80 \\
\hline $\begin{array}{l}\text { Rotineiro }=1 \\
\text { Criativo }=7\end{array}$ & 4,9 & 5,0 & 1,7 & 1 & 7 & 80 \\
\hline $\begin{array}{l}\text { Sem importância=1 } \\
\text { Importante }=7\end{array}$ & 6,3 & 7,0 & 1,1 & 1 & 7 & 81 \\
\hline
\end{tabular}

Os graus de contato com o tema diagnóstico de enfermagem, segundo as cinco atividades consideradas (leitura, aula, pesquisa, prática clínica e eventos), puderam ser analisados em termos de um único índice, já que apresentaram um alfa de Chronbach de 0,79. Dessa forma o grau de contato de cada respondente foi analisado conforme a média de contato entre as cinco atividades. O teste de Pearson resultou em 0,33 ( $p<0,001)$ mostrando fraca correlação positiva entre os escores no PDE e o grau de contato com diagnóstico.

Tabela 4. Distribuição das cargas fatoriais segundo itens e fatores. São Paulo, 2003.

\begin{tabular}{lccc}
\hline \multirow{2}{*}{ Itens } & \multicolumn{3}{c}{ Fatores e Cargas } \\
\cline { 2 - 4 } & $\mathbf{1}$ & $\mathbf{2}$ & $\mathbf{3}$ \\
\hline Significante/insignificante & $-0,854$ & 0,129 & 0,179 \\
Inválido/válido & 0,847 & $-0,151$ & $-0,098$ \\
Valioso/sem valor & $-0,770$ & 0,172 & 0,234 \\
Importante/sem importância & 0,736 & $-0,125$ & $-0,116$ \\
Relevante/irrelevante & $-0,725$ & 0,143 & 0,257 \\
Aceitável/inaceitável & $-0,703$ & 0,270 & 0,180 \\
Positivo/negativo & 0,698 & $-0,148$ & $-0,300$ \\
Conveniente/inconveniente & $-0,679$ & 0,307 & 0,327 \\
Ruim/bom & 0,672 & $-0,364$ & $-0,331$ \\
Significativo/não significativo & 0,665 & $-0,321$ & $-0,068$ \\
Inteligente/bobo & 0,657 & $-0,117$ & $-0,457$ \\
Recompensador/não compensador & 0,605 & $-0,173$ & $-0,429$ \\
Facilitador/dificultador & $-0,510$ & 0,469 & 0,130 \\
Realista/não realista & 0,432 & $-0,437$ & $-0,242$ \\
Fácil/difíil & 0,060 & 0,807 & $-0,056$ \\
Claro/ambíguo & 0,241 & $-0,695$ & $-0,136$ \\
Agradável/desagradável & $-0,330$ & 0,536 & 0,374 \\
Confortável/desconfortável & $-0,378$ & 0,528 & 0,396 \\
Criativo/rotineiro & $-0,142$ & 0,039 & 0,855 \\
\hline
\end{tabular}

Extraction Method: Principal Component Analysis Rotation Method: Varimax with Kaiser Normalization
Os respondentes enfermeiros obtiveram escore médio igual a 4,83 ( $\pm 0,92)$ e os estudantes $5,62( \pm 0,80)$. O teste de Mann-Whitney indicou que os estudantes tinham atitudes mais favoráveis que os enfermeiros $(p<0,001)$.

\section{DISCUSSÃO}

Os resultados que estimam a validade e confiabilidade do instrumento na versão brasileira indicam que as suas propriedades psicométricas são adequadas.

A correlação das médias dos escores nos 20 itens com a afirmação geral quanto ao respondente ser favorável ao diagnóstico de enfermagem mostrou resultado satisfatório (índice de correlação de Spearman =0,64; $p<0,001)$. A escala original não foi submetida a análise desse tipo, o que impede comparar o presente resultado. Na falta de "padrão-ouro" contra o qual comparar o instrumento em estudo é aceitável esse tipo de análise.

A análise de componentes principais (análise fatorial) foi realizada para acrescentar informações sobre a validade de construto do PDE. A melhor solução, com variância explicada de $61,3 \%$, mostrou que o PDE tem 3 dimensões. Em estudo com amostra de 127 enfermeiros norte-americanos ${ }^{(3)}$ a análise fatorial foi realizada por diversos métodos e os autores concluíram que 0 instrumento tem apenas uma dimensão, com $80,8 \%$ de variância explicada. Nessa publicação(3) (um resumo de trabalho apresentado em evento) os autores não apresentaram a tabela das cargas fatoriais, o que impede que os resultados entre os dois estudos sejam adequadamente comparados. Admite-se, em geral, que escalas de atitudes são unidimensionais e esse pressuposto pode ter norteado a análise fatorial do estudo norte-americano, forçando-se a solução com apenas um fator.

No presente estudo, a explicação da variância com apenas um fator atingiu percentual de $50 \%$, o que seria também aceitável. No entanto, optamos pela resolução com três fatores porque ela explica maior proporção 
da variância e também porque esse resultado é compativel com observações clássicas de Osgood ${ }^{(12)}$. Essas observações mostraram que, independentemente das diferenças de conceitos estudados e dos sujeitos cujas atitudes foram analisadas, 3 fatores ortogonais dominantes sempre reapareciam: um fator "avaliativo" (representado por itens como bom-ruim, honesto-desonesto), um fator de "potência" (representado por itens como forte-fraco, duro-macio, pesado-leve) e um fator de "atividade" (representado por itens como ativo- passivo, rápido-lento, quente-frio). Analisando-se a Tabela 4 observa-se correspondência entre os itens dos 3 fatores ali representados e os 3 fatores recorrentes nas observações de Osgood ${ }^{(12)}$. 0 fator 1 deste estudo corresponde ao fator "avaliativo" de Osgood ${ }^{(12)}$, o fator 2 ao fator de "potência" e 03 ao de "atividade". Assim, também nessa estimativa de validade, 0 PDE apresenta características adequadas.

A melhora significativa dos escores no PDE dos 100 enfermeiros participantes do curso de diagnóstico de enfermagem é mais um indicativo de validade do instrumento. Essa interpretação é possivel pelas características do curso e da sub-amostra de enfermeiros. Isto é, os resultados poderiam ter sido diferentes (ausência de diferença ou diminuição dos escores após o curso) dependendo do efeito do curso sobre a visão que os enfermeiros tinham do diagnóstico de enfermagem. Como o curso foi proposto e elaborado com a finalidade de sensibilizar os enfermeiros quanto a importância e viabilidade do diagnóstico na prática clínica, aceitou-se a suposição de melhora nas atitudes após o curso.

0 alfa de Chronbach para a amostra de 400 respondentes, com os 20 itens do PDE foi de 0,94; para a sub-amostra de 100 enfermeiros foi de 0,89 antes do curso e de 0,93 depois do curso. Em estudo norte-americano já citado $^{(3)}$ foi obtido valor de 0,97 para o alfa de Chronbach. Indicam excelente confiabilidade do instrumento.

Dos testes de associação entre o PDE e variáveis selecionadas destacase o resultado de os alunos serem mais favoráveis ao diagnóstico que os enfermeiros. A correlação (fraca) observada entre idade e atitudes é, provavelmente, efeito de o respondente ser aluno ou enfermeiro. Esse resultado era esperado e reforça a necessidade de investimentos na educação continuada dos profissionais, de forma que os estudantes possam encontrar ambientes propícios a aplicação do diagnóstico de enfermagem.

\section{CONSIDERAÇÕES FINAIS}

Estratégias que aumentem o contato dos enfermeiros e estudantes com o diagnóstico de enfermagem talvez auxiliem no desenvolvimento de atitudes mais favoráveis. Apesar de ter sido fraca, houve correlação positiva entre o grau de contato com diagnóstico de enfermagem e os escores no PDE.

Com este estudo adaptou-se para a língua portuguesa do Brasil instrumento capaz de avaliar as atitudes de enfermeiros e estudantes de enfermagem frente ao diagnóstico de enfermagem. Os testes indicaram que o instrumento adaptado, denominado em português de Posições frente ao Diagnóstico de Enfermagem, tem validade e confiabilidade adequadas para outras aplicações em nosso meio. A despeito disso, recomenda-se estimar a confiabilidade do PDE sempre que for aplicado porque a confiabilidade também depende da amostra em que o instrumento é aplicado.

\section{REFERÊNCIAS}

1. Cruz DALM. Os diagnósticos de enfermagem no ensino e na pesquisa. Rev Esc Enferm USP 1992; 26(3): 424-7.

2. Fishbein $M$, editor. Readings in attitude theory and measurement. New York (USA): Wiley \& Sons; 1967.

3. Lunney M, Krenz MA. An instrument to measure attitudes toward nursing diagnosis. In: Carroll-Johnson RM, Paquette M, editors. Classification of Nursing Diagnoses. Proceedings of the Tenth Conference of North American Nursing Diagnosis Association. San Diego (USA): Lippincott; 1992. p. 389-90.

4. Guillemin F. Cross-cultural adaptation and validation of health status measures. Scand J Reumathol 1995; 24(2): 61-3.

5. Fegert JM. Bias factors in the translation of questionnaires and classification system in international comparative child and adolescetn psychiatric research. Acta Paedopsych 1989; 29(2): 279-86.

6. Carlson E. A case study in translation methodology using the healthpromotion lifestyle profile. Pub Health Nurs 2000; 17(1): 61-70.
7. Burns N, Grove SK. The practice of nursing research: conducti, critique, and utilization. $2^{\text {nd }}$ ed. Philadelphia (USA): Saunder; 1993.

8. Nunnaly JC, Bernstein IH. Psychometric theory. $3^{\text {th }}$ ed. New York (USA): McGraw-Hill; 1994.

9. Waltz CF, Strickland OL, Lenz ER. Reliability and validity or norm-referenced. In: Waltz CF, Strickland OL, Lenz ER, editors. Measurement in nursing research. $2^{\text {nd }} \mathrm{Ed}$. ed. Philadelphia (USA): Davis; 1991. p. 161-94.

10. McDermott MAN, Palchanes K. A literature review of the critical elements in translation theory. Image 1994; 26(2): 113-7.

11. Chang AM, Chau JPG, Holroyd E. Translation of questionnaires and issues of equivalence. Jf Adv Nurs 1999; 29(2): 316-22.

12. Osgood CE. Cross-cultural comparability in attitude measurement via multilingual semantc differentials. In: Fishbein M, editor. Readings in attitude theory and measurement. New York (USA): John Wiley; 1967. p. 108-16.

\section{AGRADECIMENTOS}

Os autores agradecem a Dra. Estela Regina Ferraz Bianchi e Dra. Maria de Fátima Fernandes Vattimo pela colaboração na tradução do instrumento para o português; pela colaboração na coleta dos dados agradecemos as enfermeiras: Cristiane Giffoni Braga, Cassiana Mendes Bertoncello Fontes, Márcia Paschoalina Volpato, Suely Lopes de Azevedo e Rita de Cássia Grassia Peres. Agradecem também a Dra. Margaret Lunney e a Dra. Maryanne Krenz por terem autorizado a adaptação do instrumento e pelo suporte nos procedimentos de versão. 